\title{
Characterization of the Determinant (traB) Encoding Sex Pheromone Shutdown by the Hemolysin/Bacteriocin Plasmid pAD1 in Enterococcus faecalis
}

\author{
FloRENCE Y. AN* AND DON B. ClewElL ${ }^{*} \dagger$ \\ ${ }^{*}$ Department of Biologic and Materials Sciences, School of Dentistry, and †Department of Microbiology/ \\ Immunology, School of Medicine, The University of Michigan, Ann Arbor. Michigan 48109
}

Received June 14, 1993; revised August 6, 1993

\begin{abstract}
pADI is a hemolysin/bacteriocin plasmid originally identified in Enterococcus faecalis DSI6. It encodes a mating response to a peptide sex pheromone, cAD 1, secreted by recipient bacteria. Once pADl is acquired, production of the pheromone ceases-a trait related in part to a determinant designated $t r a B$. Here we report the nucleotide sequence of $\operatorname{tr} B$ and the position of several transposon insertions resulting in the characteristic self-induction phenotype. The deduced product has a mass of $43.7 \mathrm{kDa}$ with the C-terminal third consisting primarily of hydrophobic amino acid residues. (c) 1994 Academic Press, lnc.
\end{abstract}

Certain conjugative plasmids in Enterococcus faecalis encode a mating response to specific peptide sex pheromones secreted by potential recipient cells. (For recent reviews see Clewell, 1990, 1993a,b; Dunny, 1990). One such element is pAD1, a hemolysin/bacteriocin plasmid $(60 \mathrm{~kb})$ originally identified in the clinical isolate DS16 (Tomich et al., 1979) and representative of a large family of related hemolysin plasmids (Colomar and Horaud, 1987; Ike and Clewell, 1992). The recipient-produced pheromone, designated cADl, has been isolated and characterized (Mori et al., 1984). When pAD1 is acquired, production of the endogenous host-determined peptide is shut down. Pheromones specific for different plasmid systems, however, continue to be secreted.

The pAD1 mating response is characterized by the synthesis of a surface "aggregation substance" designated Asal (Ehrenfeld et al., 1986; Galli et al., 1989, 1990). This protein binds to the surface of recipients as well as other donors. Thus, donors exposed to culture filtrates of recipients undergo a "clumping" response, which is easily visualized and is the basis of a microtiter dilution assay for quantitating pheromone (Dunny et al., 1979). Plasmid-containing cells that have been plated on media containing CADl give rise to colonies with a characteristic "dry" morphology (Weaver and Clewell, 1988). This is related to an induced aggregation occurring within the colony.

Early studies on the regulation of the pheromone response found that transposon $(\operatorname{Tn} 917)$ insertions in two loci, designated $\operatorname{tra} A$ and $\operatorname{tra} B$, resulted in a constitutive clumping response (Ike and Clewell, 1984). These derivatives also transfer plasmid DNA in short (e.g., $10 \mathrm{~min}$ ) matings at frequencies much higher (e.g., over a 1000 -fold) than the wildtype, which normally takes well over 20 min to become fully induced. traA mutants exhibited the dry colony morphology on solid media in the absence of pheromone. The product of this determinant has been shown to represent a key negative regulator of $\operatorname{tr} a E 1$, whose product in turn positively regulates all or most of the structural genes related to conjugation (Clewell and Weaver, 1989; Ehrenfeld and Clewell, 1987; Galli et al., 1992; Pontius and Clewell, 1992a, 1992b; Tanimoto and Clewell, 1993; Weaver and Clewell, 1988, 1989, 1990).

In contrast to the case for traA mutations, colonies of $\operatorname{traB}$ derivatives had a "ringed" morphology on plates without pheromone; 
these corresponded to a dry area in the middle of the colony with the outside portion (i.e., the ring) having a wildtype appearance (Weaver and Clewell, 1991). The traB determinant is believed to encode a product that is involved in shutdown of endogenous cAD1 production, although additional factors are also involved (Weaver and Clewell, 1990, 1991). Culture filtrates of $t r a B$ mutants have barely detectable amounts of cAD1, but enough to result in self induction. The ring appearance of colonies is probably due to the fact that pheromone production is affected by oxygen (Weaver and Clewell, 1991), with higher levels of cAD1 being produced (as much as 16 -fold more) by cells in a more anaerobic environment. Since the center of the colony is more anaerobic, more pheromone is produced resulting in self aggregation.

The regulatory region for the $\mathrm{pAD} 1$ pheromone response is organized as -traEl-iadtraA-traC-traB [see Fig. 1 (repA, repB, and rep $C$ in Fig. 1 represent determinants relating to replication and maintenance)]. iad (Clewell et al., 1990) is the determinant for a peptide $\mathrm{ADD} 1$ which is a competitive inhibitor of CAD1, whereas $t r a C$ encodes a surface (extracellular) protein involved in binding pheromone and ADD1 (Weaver and Clewell, 1988; Tanimoto et al., 1993). All but traA are transcribed right to left. The nucleotide sequence of all but $\operatorname{tr} a B$ has been reported elsewhere (Clewell et al., 1990; Pontius and Clewell, 1992a,b; Tanimoto et al., 1993; Weaver et al., 1993). Here we report the sequence of $\operatorname{tr} a B$ and identify the precise location of specific mutational lesions. Where not specifically noted or cited, details of cloning, nucleotide sequence determination, introduction of plasmids into bacteria by conjugation or electroporation, and other aspects of the methodology were essentially as previously described (Ausubel, et al., 1987; Cruz-Rodz and Gilmore, 1990; Pontius and Clewell, 1992a; Su et al., 1991; Wirth et al., 1986).

The sequence was determined (both strands) using derivatives (nested deletions) of the Escherichia coli DH5 $\alpha$ clones pAM7500, pAM2603, and pAM3101 indicated in Fig. 1. The vector pBluescript SK + II was utilized in the case of pAM7500 and pAM3101, whereas pBluescript KS+ II was involved in the case of pAM2603 (Pontius and Clewell, 1992a; Weaver et al., 1993). In some cases synthetic oligonucleotides served as primers in sequencing reactions. The location of Tn917lac insertions was determined using primers that direct synthesis out the ends of the transposon (Pontius and Clewell, 1992a).

Computer analysis revealed a large open reading frame $(\mathrm{ORF})^{1}$ (Fig. 2) starting with ATG and corresponding to a protein with 388 amino acid residues with a molecular weight of 43,687 and a $\mathrm{p} I$ value of 9 . A good potential ribosome binding site (GGAGG) is located eight nucleotides upstream. Although an in-frame ATG is located 5 "codons" upstream, the absence of a related ribosome binding site implies that it is not a functional translational start site. The only other reading frames detected corresponding to greater than 25 amino acid residues were on the opposite strand and had no related ribosome binding sites. [They were located within the above-noted ORF and corresponded to 29 and 133 residues (not shown).] The positions of five previously reported (Weaver and Clewell, 1988) Tn917lac insertions resulting in the $\operatorname{tr} B$ phenotype were found here to be spread through a significant portion of the large ORF (Figs. 1 and 2). It was therefore concluded that the ORF corresponded to $\operatorname{tra} B$. The $5^{\prime}$ end was 46 bp downstream from the 3 terminus of rep $A$ (Weaver et al., 1993); and the $3^{\prime}$ end was $29 \mathrm{bp}$ from the $5^{\prime}$ end of $t$ raC (Tanimoto et al., 1993). A potential sigma-70-type promoter sequence is located upstream from the start codon, and a 16-base region with dyad symetry is located between the promoter and ribosome binding site. It is conceivable that the latter is a target for a regulatory protein, although there is currently no evidence that $\mathrm{TraB}$ is regulated. The absence of any obvious transcriptiontermination site near the $3^{\prime}$ region of $\operatorname{traB}$ suggests that transcription might extend into

\footnotetext{
${ }^{1}$ Abbreviation used: ORF, open reading frame.
} 

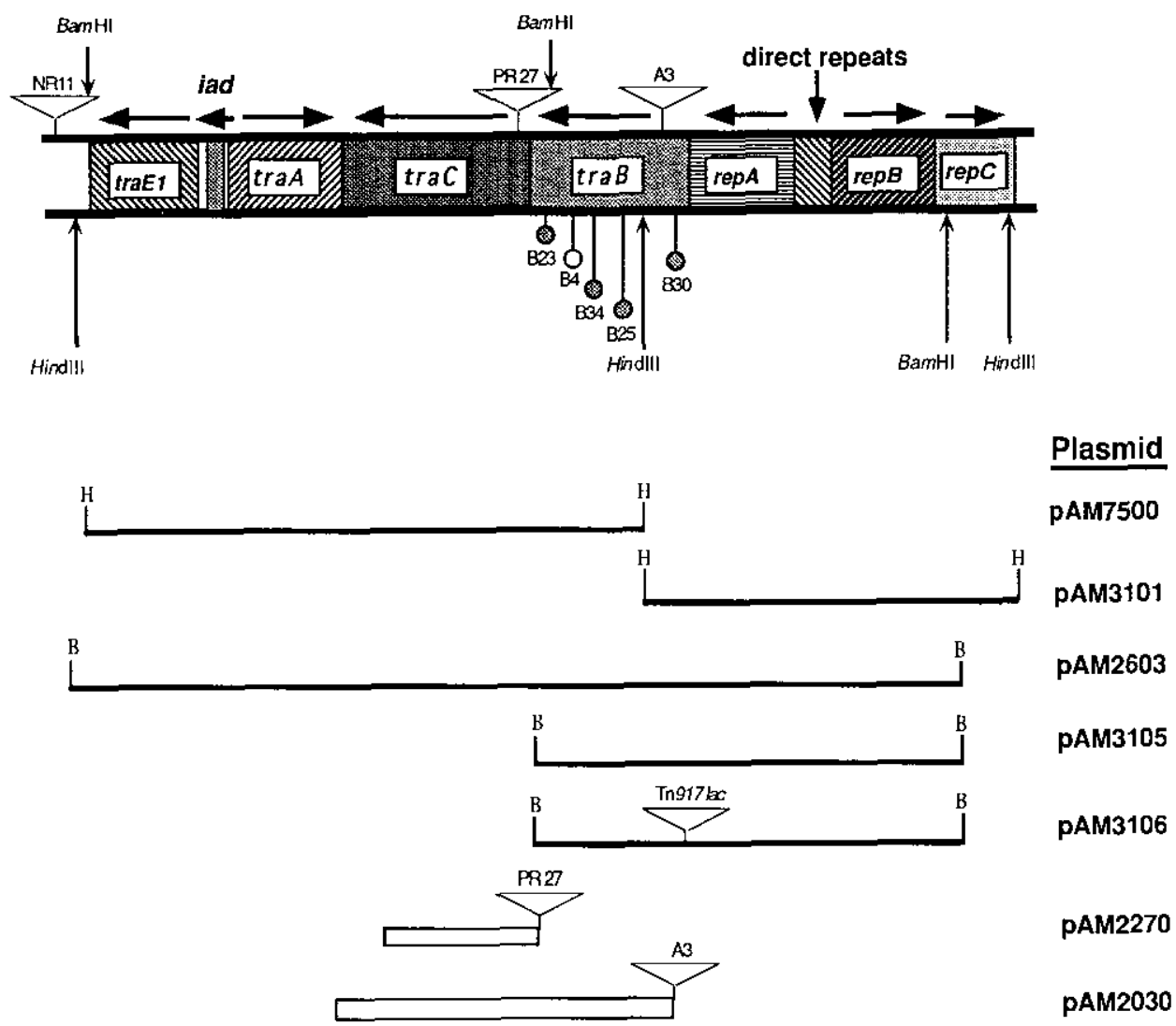

FIG. 1. Map of pADI region carrying $\operatorname{raB}$ and flanking genes. The arrows reflect $5^{\prime}$ to $3^{\prime}$ orientation above the labeled genes. The filled circles (B23, B34, B25 and B30) represent insertions of Tn 917 lac in $t$ raB which are oriented such that $\mathrm{LacZ}$ is expressed under the transcription of $t \mathrm{raB}$. The open circle, representing B4, corresponds to an insertion in the opposite orientation. The triangles corresponding to NR 11 (see Weaver and Clewell, 1988), PR27, and A3 are also Tn917lac insertions with orientations similar to those of the filled circles. [pAM2270 (PR27) and pAM2030 (A3) represent deletions involving the indicated regions.] The location of certain restriction sites used in the cloning efforts is also shown. (H and B refer to HindIII and BamHI sites, respectively.) Cloned segments (noted for pAM7500, pAM3101, and pAM2603) in the plasmid vector pBluescript $(E . c o l i$ DH $5 \alpha$ ) were used for DNA sequencing analyses. The plasmids, pAM3105 and pAM3106, were derivatives of the $E$. coli-E. faecalis shuttle plasmid pAM40I (Wirth $e t$ al., 1986) carrying the noted segment. (The left-end BamHI sites correspond to sites in the transposon inserted in PR27.) The transposon insertion in pAM 3106 is the same as that of B30 and was generated via allelic exchange by first introducing pAM2300 into OG1X(pAM3105) by conjugation, selecting on both chloramphenicol (for the vector marker) and erythromycin (for the transposon marker on pAM2300), and then using a plasmid preparation from a resulting transconjugant to transform a plasmid free OG!X strain selecting for erythromycin and chloramphenicol resistance and screening for absence of hemolysin expression. The plasmids pAM2270 and pAM2030 are derivatives of pAD1 carrying the designated Tn917/ac insertions (PR27 or A3) with the adjacent deletions noted.

traC. A high degree of hydrophobicity in the C-terminal third of the deduced TraB suggests an association of this region with the membrane.

The orientation of the Tn917lac insertions corresponding to B30 (pAM2300), B25 (pAM2250), B34 (pAM2340), and B23
(pAM2230) (see Figs. 1 and 2) are consistent with their giving rise to light blue colonies on plates containing $\mathrm{X}$-gal, reflecting the low constitutive expression of TraB from a promoter upstream of the $5^{\prime}$ end; cells carrying the oppositely oriented B4 (pAM2040) gave rise to white colonies (Weaver and Clewell, 


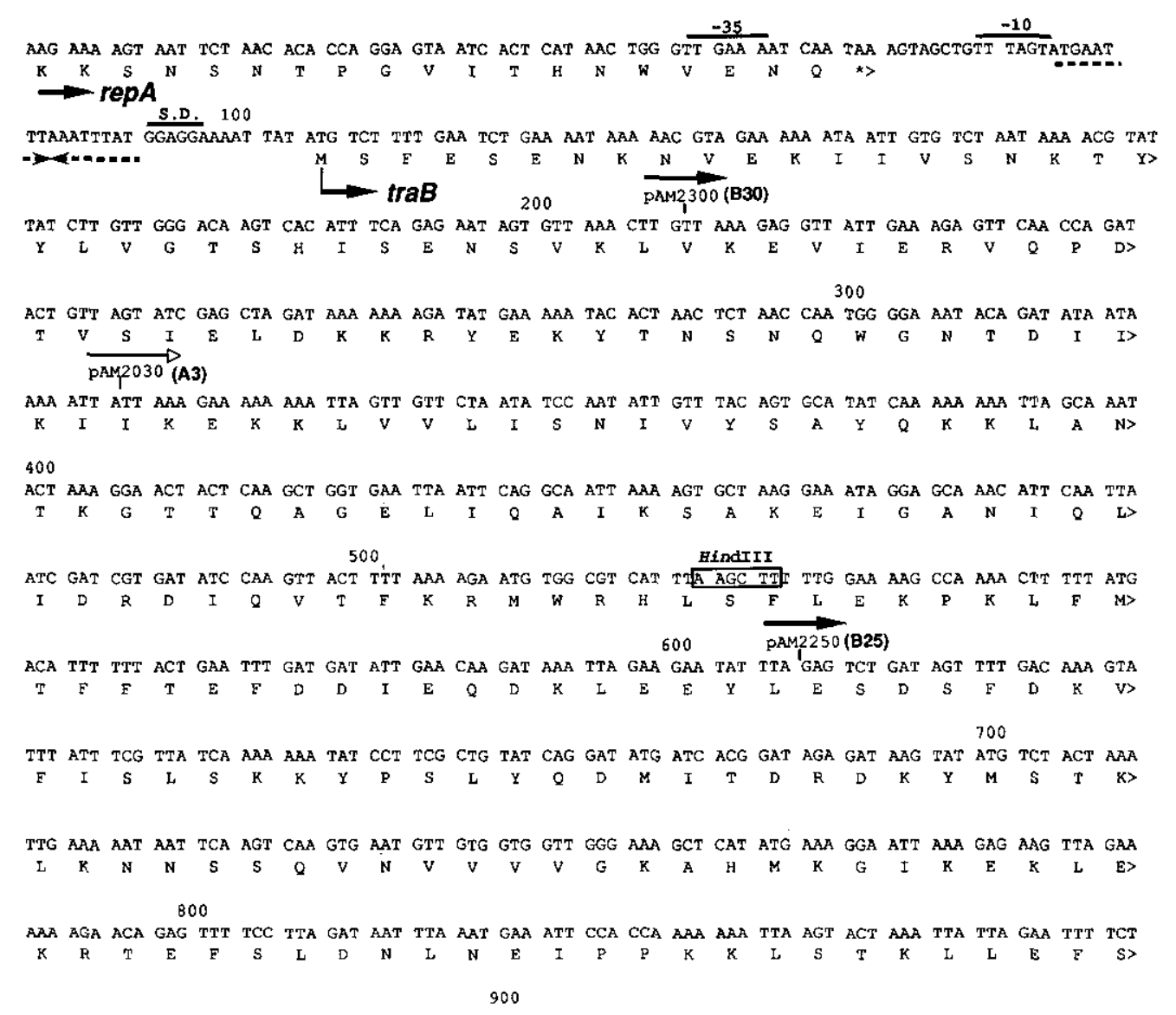

FIG. 2. Nucleotide sequence of region of pADI containing $\operatorname{tr} a B$ and portions of flanking determinants. To view the determinant in the more convenient $5^{\prime}$ to $3^{\prime}$ orientation, the sequence is reversed compared to that of Fig. 1. The position of the various Tn9 7 /ac insertions, with their orientations indicated relative to the ability of LacZ to be expressed via transcription of $\operatorname{tr} a B$, and the names of the corresponding pAD1 derivatives are indicated. The positions of potential ribosome binding sites [S.D. (Shine and Dalgarno, 1975)] and sigma-70-like promoters ( -10 and -35 hexamers) are overlined. The dashed arrows reflect a region of dyad symetry just upstream of $\operatorname{tr} B$. The GenBank accession number for the sequence is $\mathrm{U} 00681$.

1988, 1990). The position of two insertions, A3 and PR27, which were associated with deletions (Clewell and Weaver, 1988; Weaver and Clewell, 1988, 1990), was also determined by sequence analysis (Figs. 1 and 2). The A3 derivative corresponding to pAM2030 was deleted for most of $t \mathrm{raB}$, all of $\mathrm{traC}$, and through the codon for the eighth amino acid residue in from the C-terminus of $\operatorname{traA}$. The deletion corresponding to PR27 (pAM2270) is actually located downstream of $\operatorname{traB}$ and completely within $\operatorname{traC}$. It begins at $\mathrm{TraC}$ amino acid residue 60 and ends with residue 384. LacZ expression in both the A3 and
PR27 derivatives was constitutive at a low level (Weaver and Clewell, 1988). A3 was shown previously to exhibit the traA phenotype, whereas PR27 was a "partiâl responder" to pheromone (Weaver and Clewell, 1988).

The plasmids pAM3105 and pAM3106 (Fig. 1) represent clones of traB-containing DNA segments in the E. coli-E. faecalis shuttle vector pAM401 (Wirth et al., 1986). Culture filtrates of late exponential phase $E$. faecalis OG1X cells carrying pAM3105 showed a pheromone (cAD1) titer of 8 , compared to 256 for a filtrate of cells harboring 


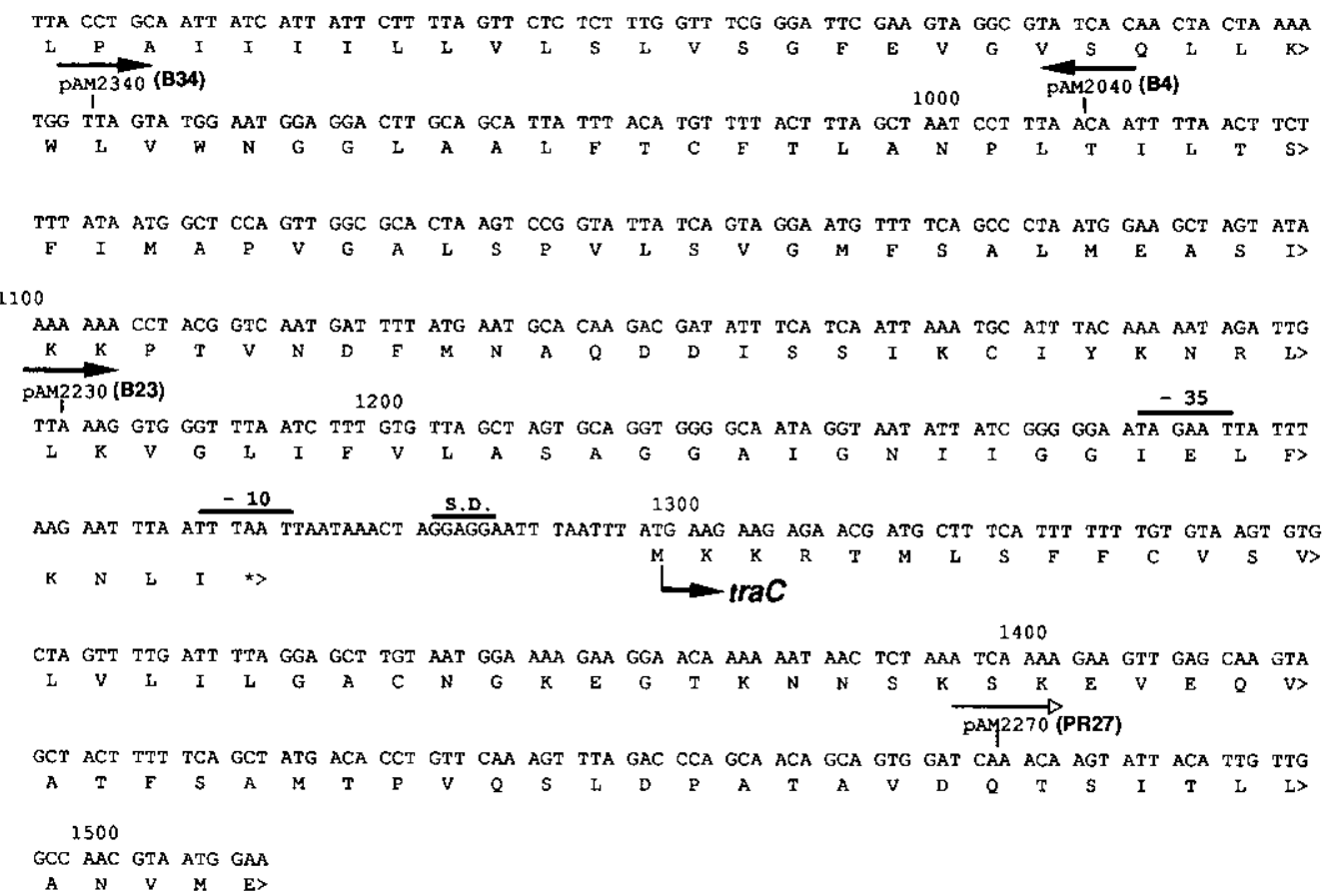

FIG. 2-Continued

just the vector. As expected, in the case of pAM3106, which has a transposon insertion in $\operatorname{traB}$, the titer was the same as for the vector alone. Consistent with these results is the observation that pAM3105 appears to complement, in trans, the traB phenotype associated with pAM2300 (Tn917lac traB insertion $\mathrm{B} 30$ in pAD1; see Fig. 2). OG1X(pAM2300) exhibited the "ringed colony" phenotype on solid media, whereas OG1X(pAM2300, pAM3105) colonies appeared similar to wildtype pAD1 (data not shown). Homologous recombination was not ruled out unambiguously here since the host was not recombination-deficient; however, plasmid isolation and analyses using $E c o$ RI and $B a m H I$ revealed the presence of the two plasmids at their expected sizes. [The only E. faecalis Rec-deficient host available is UV202 (Yagi and Clewell, 1980), a nonisogenic host unable to support a pAD1 pheromone response (Ike and Clewell, 1984) and therefore not useful for the above test.]

The transposon-generated deletions associated with PR27 (carries pAM2270) and A3 (carries pAM2030) (Figs. 1 and 2) were previously reported (Weaver and Clewell, 1988) to give rise to titers of $<2$ and 64 ; these values were confirmed here when determined in experiments done in parallel with those involving pAM3105 and pAM3106 (see above). In the case of pAM2030, the observed apparent fourfold reduction (i.e., from 256 down to 64 ), even though a significant portion of $\operatorname{tra} B$ was missing, is believed due to the associated dysfunction of $t r a C$. The $t r a C$ phenotype is generally characterized by a fourfold increase in IAD1 in culture filtrates due to a reduction in peptide binding (Weaver and Clewell, 1988); this results in a masking of the cAD1 titer.

Figure 3 shows a dot matrix plot demonstrating significant similarity between $\operatorname{traB}$ and a determinant on the pheromone-responding plasmid pCF10 designated prgY (Ruhfel et al., 1993). The size of the gene products are very similar and, like the case for TraB, the putative $\operatorname{prg} Y$ product exhibits strong hydrophobicity in the C-terminal third of its structure. Significant homology 


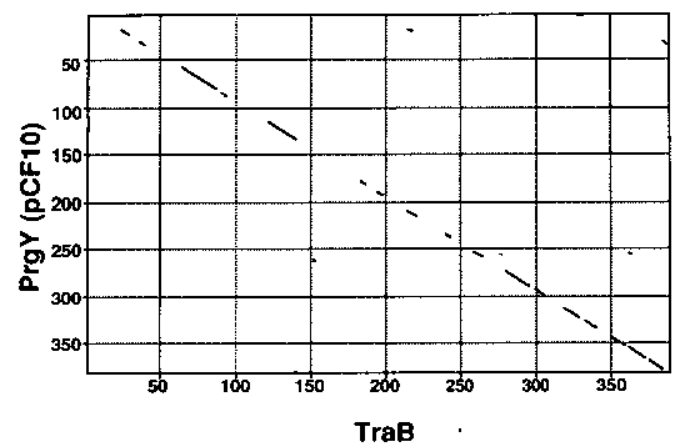

FIG. 3. Homology matrix plot comparing TraB with PrgY of pCF 10. A MacVector (IBI) program was used to generated the comparisons. Each point reflects at least $60 \%$ homology within an eight amino acid alignment.

with other sequences in the GenBank data base was not detected.

It is not known how many sex pheromones are secreted by plasmid-free strains of $E$. faecalis. There are at least five and probably many more (see Clewell, 1993b). It has been speculated (Clewell, 1981, 1985) that these peptides may originally have had, and perhaps continue to have, different functions and that plasmids evolved to take advantage of them as mating signals. Since a given plasmid generally shuts down production of only the corresponding pheromone, its related determinant(s) must be specific. The homology shared by $\operatorname{tra} B$ and a pCF 10 reading frame $\operatorname{prg} Y$ suggests that the latter might be involved with shutdown of endogenous CCF10 (Ruhfel et al., 1993); however, there is no functional evidence yet for such a role. The two determinants appear to have evolved from a common ancestor. It is noteworthy that $\mathrm{pAD} 1$ and pCF 10 also exhibit significant homology with respect to genes determining aggregation substance (Galli et al., 1990; Kao et al., 1991), surface exclusion (Kao et al., 1991; Weidlich et al., 1992), and pheromone binding (Ruhfel et al., 1993; Tanimoto et al., 1993).

The nature of the shutdown process is not known. Indeed, genetic determinants for enterococcal sex pheromones have not yet been identified, and thus nucleotide sequence information that might provide clues regarding their regulation is not available.

\section{ACKNOWLEDGMENTS}

We thank K. Tanimoto for pAM2603 and L. Pontius for pAM3101. We also thank K. Tanimoto and G. Dunny for helpful discussions and $G$. Dunny for sharing the amino acid sequence of PrgY prior to publication. This study was supported by Public Health Service Grant GM33956 from the National Institutes of Health.

\section{REFERENCES}

Ausubel, F. M., Brent, R., Kingston, R. E., Moor, D. D., Seidman, J. G., Smith, J. A., ANd Struhl, K. (1987). "Current Protocols in Molecular Biology." Wiley, New York.

Clewell, D. B. (1981). Plasmids, drug resistance, and gene transfer in the genus Streptococcus. Microbiol. Rev. 45, 409-436.

Clewell, D. B. (1985). Sex pheromones, plasmids, and conjugation in Streptococcus faecalis. In "The Origin and Evolution of Sex. MBL Lecture Series in Biology, Vol. 7" H. O. Halvorson and A. Monroy, Eds.), pp. 13-28. A. R. Liss, New York.

Clewell, D. B. (1990). Movable genetic elements and antibiotic resistance in enterococci. Eur. J. Clin. Microbiol. Infect. Dis. 9, 90-102.

Clewell, D. B. (1993a). Bacterial sex pheromone-induced plasmid transfer. Cell 73, 9-12.

Clewell, D. B. (1993b). Sex pheromones and the plasmid-encoded mating response in Enterococcus faecalis. In "Bacterial Conjugation" (D. B. Clewell, Ed.), pp. 349-367. Plenum Press, New York.

Clewell, D. B., Pontius, L. T., AN, F. Y., IKe, Y., Suzukl, A., AND NAKAyama, J. (1990). Nucleotide sequence of the sex pheromone inhibitor (iAD1) determinant of Enterococcus faecalis conjugative plasmid pAD1. Plasmid 24, 156-161.

Clewell, D. B., and Weaver, K. E. (1989). Sex pheromones and plasmid transfer in Enterococcus faecalis. Plasmid 21, 175-184.

Colomar, 1., AND Horaud, T. (1987). Enterococcus faecalis hemolysin-bacteriocin plasmids belong to the same incompatibility group. Appl. Environ. Microbiol. 53, 567-570.

Cruz-Rodz, A. L., ANd Gilmore, M. S. (1990). High efficiency introduction of plasmid DNA into glycine treated Enterococcus faecalis by electroporation. $\mathrm{Mol}$. Gen. Genet. 224, 152-154.

DunNY, G. M. (1990). Genetic functions and cell-cell interactions in the pheromone-inducible plasmid transfer system of Enterococcus faecalis. Mol. Microbiol. 4, 689-696.

Dunny, G. M., Craig, R. A., Carron, R. L., AND CleWELL, D. B. (1979). Plasmid transfer in Streptococcus faecalis: Production of multiple sex pheromones by recipients. Plasmid 2, 454-465.

EHRENFELD, E. E., AND Clewell, D. B. (1987). Transfer functions of the Streptococcus faecalis plasmid pAD1: organization of plasmid DNA encoding response to sex pheromone. J. Bacteriol. 169, 3473-3481. 
EhrenFeld, E. E., Kessler, R. E., AND Clewell, D. B. (1986). Identification of pheromone-induced surface proteins in Streptococcus faecalis and evidence of a role for lipoteichoic acid in formation of mating aggregates. J. Bacteriol. 168, 6-12.

Galli, D., Friesenegger, A., AND WIRTH, R. (1992). Transcriptional control of sex-pheromone-inducible genes on plasmid pADI of Enterococcus faecalis and sequence analysis of a third structural gene for (pPD1encoded) aggregation substance. Mol. Microbiol. 6, 1297-1308.

Galli, D., LotTspeich, F., AND Wirth, R. (1990). Sequence analysis of Enterococcus faecalis aggregation substance encoded by the sex pheromone plasmid pAD1. Mol. Microbiol. 4, 895-904.

Galli, D., WirTh, R., AND WANNER, G. (1989). Jdentification of aggregation substances of Enterococcus faecalis cells after induction by sex pheromones-An immunological and ultrastructural investigation. Arch. Microbiol. 151, 486-490.

IKE, Y., AND CLEWELL, D. B. (1984). Genetic analysis of the pAD 1 pheromone response in Streptococcus faecalis, using transposon $\mathrm{Tn} 917$ as an insertional mutagen. J. Bacteriol. 158, 777-783.

IKE, Y., AND Clewell, D. B. (1992). Evidence that the hemolysin/bacteriocin phenotype of Enterococcus faecalis subsp. zymogenes can be determined by plasmids in different incompatibility groups as well as by the chromosome. J. Bacteriol. 174, 8172-8177.

KaO, S., Olmsted, S. B., Viksnins, A. S., Gallo, J. C., AND DunNy, G. M. (1991). Molecular and genetic analysis of a region of the plasmid $\mathrm{pCF} 10$ containing positive control genes and structural genes encoding surface proteins involved in pheromone-inducible conjugation in Enterococcus faecalis. J. Bacteriol. 173, 7650-7664.

Mori, M., SaKagami, Y., Narita, M., Isogal, A., Fujino, M., Kitada, C., Craig, R., Clewell, D. B., AND SuZUKI, A. (1984). Isolation and structure of the bacterial sex pheromone, cADI, that induces plasmid transfer in Streptococcus faecalis. FEBS Lett. 178, 97100.

Pontius, L. T., AND Clewell, D. B. (1992a). Regulation of the pADl-encoded pheromone response in $\mathrm{En}$ terococcus faecalis: Nucleotide sequence analysis of traA. J. Bacteriol. 174, 1821-1827.

Pontius, L. T., AND Clewell, D. B. (1992b). Conjugative transfer of Enterococcus faecalis plasmid pAD1: nucleotide sequence and transcriptional fusion analysis of a region involved in positive regulation. $J$. Bacteriol. 174, 3152-3160.

Ruhfel, R. E., Manias, D. A., and Dunny, G. M. (1993). Cloning and characterization of a region of the Enterococcus faecalis conjugative plasmid, pCF10, encoding a sex pheromone binding function. $J$. Bacteriol. 175, 5253-5259.

Shine, J., AND DALGaRno, L. (1975). The $3^{\prime}$ terminal sequence of Escherichia coli $16 \mathrm{~S}$ ribosomal RNA:
Complementary to nonsense triplets and ribosome binding sites. Proc. Natl. Acad. Sci. USA 71, 13421346.

Su, Y. A., Sulavik, M. C., He, P., Makinen, K., MaKINEN, P., FIEDLER, S., WirTh, R., AND CleWEll, D. B. (1991). Nucleotide sequence of the gelatinase gene (gelE) from Enterococcus faecalis subsp. liquefaciens. Infect. Immun. 59, 415-420.

TANimoto, K., AN, F. Y., AND Clewell, D. B. (1993). Characterization of the $t \mathrm{raC}$ determinant of the Enterococcus faecalis hemolysin/bacteriocin plasmid pAD1. Binding of sex pheromone. $J$. Bacteriol. 175, 5260-5264.

TANimoto, K., AND Clewell, D. B. (1993). Regulation of the pADl-encoded sex pheromone response in Enterococcus faecalis: Expression of the positive regulator TraE1. J. Bacteriol. 175, 1008-1018.

TOMICH, P. K., AN, F. Y., DAMLE, S. P., AND ClEWEll, D. B. (1979). Plasmid related transmissibility and multiple drug resistance in Streptococcus faecalis subsp. zymogenes strain DS16. Antimicrob. Agents Chemother. 15, 828-830.

WEAVER, K. E., AND ClewEll, D. B. (1988). Regulation of the pAD1 sex pheromone response in Enterococcus faecalis: Construction and characterization of lacZ transcriptional fusions in a key control region of the plasmid. J. Bacteriol. 170, 4343-4352.

Weaver, K. E., and Clewell, D. B. (1989). Construction of Enterococcus faecalis pAD1 miniplasmids: Identification of a minimal pheromone response regulatory region and evaluation of a novel pheromone-dependent growth inhibition. Plasmid 22, 106-119.

WEAVER, K. E., AND Clewell, D. B. (1990). Regulation of the pAD1 sex pheromone response in Enterococcus faecalis: Effects of host strain and traA, traB, and $\mathrm{C}$ region mutants on expression of an $\mathrm{E}$ region pheromone-inducible lacZ fusion. $J$. Bacteriol. 172, 26332641.

Weaver, K. E., AND Clewell, D. B. (1991). Control of Enterococcus faecalis sex pheromone cAD1 elaboration: Effects of culture aeration and pADI plasmid-encoded determinants. Plasmid 25, 177-189.

Weaver, K. E., Clewell, D. B., AND AN, F. Y. (1993). Identification, characterization, and nucleotide sequence of a region of Enterococcus faecalis pheromone-responsive plasmid pAD1 capable of autonomous replication. J. Bacteriol. 175, 1900-1909.

WeIDLICH, G., WirTh, R., AND Galli, D. (1992). Sex pheromone plasmid pADl-encoded surface exclusion protein of Enterococcus faecalis. Mol. Gen. Genet. 233, $161-168$.

WiRTH, R., AN, F. Y., AND Clewell, D. B. (1986). Highly efficient protoplast transformation system for Streptococcus faecalis and a new Escherichia coli-S. faecalis shuttle vector. J. Bacteriol. 165, 831-836.

YAGI, Y., AND CLEWELL, D. B. (1980). Recombinationdeficient mutant of Streptococcus faecalis. J. Bacteriol. 143, 966-970.

Communicated by Francis L. Macrina 